\title{
Administration of care to older patients in transition from hospital to home care services: home nursing leaders' experiences
}

This article was published in the following Dove Press journal:

Journal of Multidisciplinary Healthcare

3 October 2013

Number of times this article has been viewed

Bjørg Dale'

Sigrun Hvalvik ${ }^{2}$

'Centre for Caring Research Southern Norway, Faculty of Health and Sport Sciences, University of Agder, Grimstad, ${ }^{2}$ Centre for Caring Research - Southern Norway, Faculty of Health and Social Studies, Telemark University College, Porsgrunn, Norway
Correspondence: Bjørg Dale

Centre for Caring Research - Southern Norway, Faculty of Health and Sport

Sciences, University of Agder, PO Box 509, NO-4898 Grimstad, Norway

$\mathrm{Tel}+4737233752$

Email bjorg.dale@uia.no
Background: Older persons in transition between hospital and home care services are in a particularly vulnerable situation and risk unfortunate consequences caused by organizational inefficiency. The purpose of the study reported here was to elucidate how home nursing leaders experience the administration of care to older people in transition from hospital to their own homes.

Methods: A qualitative study design was used. Ten home nursing leaders in two municipalities in southern Norway participated in individual interviews. The interview texts were audio taped, transcribed verbatim and analyzed by use of a phenomenological-hermeneutic approach.

Results: Three main themes and seven subthemes were deduced from the data. The first main theme was that the home nursing leaders felt challenged by the organization of home care services. Two subthemes were identified related to this. The first was that the leaders lacked involvement in the transitional process, and the second was that they were challenged by administration of care being decided at another level in the municipality. The second main theme found was that the leaders felt that they were acting in a shifting and unsettled context. Related to this, they had to adjust internal resources to external demands and expectations, and experienced lack of communication with significant others. The third main theme identified was that the leaders endeavored to deliver care in accordance with professional values. The two related subthemes were, first, that they provided for appropriate internal systems and routines, and, second, that they prioritized available professional competence, and made an effort to promote a professional culture.

Conclusion: To meet the complex needs of the patients in a professional way, the home nursing leaders needed to be flexible and pragmatic in their administration of care. This involved utilizing available professional competence appropriately. The coordination and communication between the different organizational levels and units were pointed out as major factors requiring improvement.

Keywords: cooperation, geriatric patients, home nursing leaders, phenomenological-hermeneutic method, prioritization, transitional care

\section{Introduction}

Home nursing leaders, managers, and clinicians are uniquely placed to address the challenges and capitalize on the opportunities to ensure that older patients in transition from hospital to their own home receive proper care and treatment., ${ }^{1,2}$ Insight into how home nursing leaders experience their abilities to administer quality care for this group of patients is therefore of great interest. Home nursing leaders are part of a wider community care system. Their responsibilities are complex and multifaceted, comprising personnel management, budget management, as well as day-to-day 
patient care. Home nursing leaders are jointly responsible for the coordination and quality of care provided to patients in transition from hospital to home.

The number of older patients who are discharged from hospital to their homes and still in need of professional care is increasing in Norway. One of the main reasons for this is the Coordination Reform, ${ }^{3}$ which took effect in January 2012. A major ambition with this reform is to increase and improve the coordination between the hospitals and the municipalities, and to further develop the health care services in the municipalities. A significant aim is the quick return of patients to the home municipality after treatment in the specialist health care services. ${ }^{3}$ Consequently, patients are now discharged from hospital sicker, more dependent on care, and at higher risk of readmission than before the reform was introduced. ${ }^{4,5}$ In the transfer from one care setting to another, a range of problems may arise, such as limited clinical information flow, insufficiently updated care plans, and medication-related errors. ${ }^{6}$ These problems not only contribute to increases in rates of readmission and economic costs; the problems also affect the patients' lives and personal transition process, and challenge those who are further responsible for the quality of care the patients receive.

The transfer of patients from hospital to home can be associated with the term "transitional care." According to Naylor et al, "transitional care" refers to a range of timelimited services and environments designed to ensure health care continuity and avoid preventable poor outcomes among at-risk populations as they move from one level of care to another, among multiple providers, and/or across settings. Quality in transitional care is particularly important for vulnerable patient groups coping with multiple chronic conditions and complex medication regimens. ${ }^{1,8}$ Older patients in transition from hospital to their homes can be characterized as one such group. While being in transitional care, the patients are at the same time situated in a personal transition process related to health and illness, often involving loss and challenges that are undesired. ${ }^{9}$ The transitional care and the transition process can thus be considered intertwined and interacting. One goal in nursing from a transition perspective is to facilitate healthy transition processes. ${ }^{10}$ The administration of care that provides healthy transitions for vulnerable older patients who are discharged from hospital to their own homes entails extensive responsibilities. These responsibilities are to be dealt with in a context characterized by increased demands on time and cost efficiency, improved patient outcomes, and decreased length of hospital stay. ${ }^{2,11-13}$ The home care services in Norway are organized following the so-called purchaser-provider model, inspired by the New Public Management movement. ${ }^{12}$ This model implies that the responsibility for assessing and designing a contract for service provision should be separated from the responsibility for providing the services. ${ }^{12}$ The responsibility for needs assessment is accordingly placed within the purchaser unit, which is a separate unit in the municipality health care services. They cooperate with the home nursing units in the municipality, who are expected to carry out the care and to reassess the patients' care needs if necessary.

Transition of patients between levels of care has been recognized as an important area for improvement in health care quality and patient safety on an international level for several years. ${ }^{6,11}$ Studies related to this subject have particularly focused on hospital discharge of older patients, readmission, and return to hospital. ${ }^{14-17}$ Effective communication and information have been pointed out as vital in several areas in these studies, within and between levels of care as well as between the health care providers and the patients and the family caregivers. In a review study focusing on transitional care for older adults, Naylor ${ }^{18}$ found that older patients and their caregivers reported substantial unmet needs. The included studies also demonstrated that differences in expectations between patients, their families, and health care providers, as well as the need for increased patient and family involvement in decision making, were common themes in the discharge process.

Transitional care intervention models provide comprehensive discharge planning and home follow-up care for chronically ill, high-risk, older adults hospitalized for common medical and surgical conditions. ${ }^{2}$ Multiple studies have been designed to test nurse-led as well as multidisciplinaryled models, and some demonstrate capacity to improve health outcomes and reduce health costs. ${ }^{19,20}$ In a review study, Naylor et $\mathrm{al}^{21}$ examined transitional care models and interventions in relation to effect on hospital readmission, and revealed that nearly a dozen interventions demonstrated some positive impact on preventing hospital readmissions.

Norwegian studies focusing on the transitional care from a nursing perspective seem to be sparse. One exception is a study performed by Danielsen and Fjær. ${ }^{22}$ They interviewed hospital nurses and nurses from the municipality to explore their experiences related to the transfer of older patients from the hospital to the municipality. They found that various practical problems related to communication represented a potential danger for risky transitions. The need for improved knowledge about organizational conditions on both levels was revealed, as well as knowledge about the patient's condition and care 
needs. Home care nurses' efforts and ambitions to provide excellent care for older patients in transition have been shown to be challenged and threatened by various circumstances in the caring context. ${ }^{23}$ Cooperation across organizational levels was pointed out as one critical factor with potential for improvement. ${ }^{23}$ However, national as well as international research focusing on home care nursing leaders' own experiences and perceptions regarding the administration of care to patients in transition in general, and from hospital to home in particular, seem to be limited. Therefore, the aim of this study was to elucidate how home nursing leaders experienced the administration of care to older people in transition from hospital to their own homes.

\section{Methods}

\section{Participants}

A purposive sampling method was used to recruit participants for this study. The home care offices in two municipalities in two different counties in southern Norway were contacted and informed about the project, and home nursing leaders were invited to participate in individual interviews. This invitation resulted in ten nursing leaders, five from each municipality, agreeing to participate. They were all female and trained nurses, and their experience as home nursing leaders ranged from 1 to 10 years' duration.

\section{Data collection}

To gain insight and a deeper understanding of the nursing leaders' lived experiences of the transition process for older patients from hospital to home, individual interviews were undertaken for data collection. A semi-structured interview guide directed the interviews, which were carried out in accordance with common recommendations. ${ }^{24,25}$ Initially, the participants were asked to discuss, in general and freely, their lived experiences concerning patients who are in transition to receive professional home care. As the participants all focused on their experiences related to the transition from hospital to home, the researchers decided to follow-up this specific transition process.

The interview guide included themes such as organization of the care services, internal and external cooperation, personnel resources, routines, social milieu, and values that guided the attitudes and quality of care services. As these themes were spontaneously mentioned by the participants, the researchers merely had to follow-up and encourage them to further expand on and deepen their views.

Both authors of this paper were involved in the interview process. They had a basic understanding of the phenomenon under investigation, as they were familiar with the actual practice field as nurses and nursing teachers. Consequently, this eased the process of asking relevant follow-up questions. Before the interviews started, the researchers thoroughly discussed and reflected on the subject for the interviews, which was important to ensure a consistent interview approach.

Both authors were equally involved in all phases of the study, including transcription and analyses of the interview texts. The interviews were conducted in the informants' offices or in a private room at the researchers' work place during May to August 2012. They lasted between 40 and 60 minutes. In agreement with the participants, the interviews were audio taped and subsequently transcribed verbatim.

\section{Data analysis}

During the analyzing process, the two researchers worked both separately and together. Their interpretations were compared, discussed, and reflected on. Both authors analyzed the interview text, and a phenomenological-hermeneutic approach was used to understand and interpret the meaning of the lived experiences as narrated by the home nursing leaders in this study. The method was developed by Lindseth and Norberg ${ }^{26}$ and grounded in the philosophical assumptions of Paul Ricoeur. ${ }^{27}$ When using a phenomenological-hermeneutic method for analyzing a text, the researcher continuously moves back and forth between the parts and the whole of the text. The interpretation of a text starts, according to Ricoeur, ${ }^{27}$ with grasping the meaning of the text as a whole - that is, what the text is about. ${ }^{28}$ After that initial phase, the researcher seeks to gain a deeper understanding of the relationship between the whole and the parts of the text by entering the hermeneutic circle and, at the same time, continuously moving forward. ${ }^{26}$ In this sense, the method provides a dialectic movement between understanding and explanation, ${ }^{26}$ and understanding and interpretation. ${ }^{28}$ According to Ricoeur, ${ }^{27}$ a text lives its own life in the sense that it should be objectified and separated from the narrator. The process also includes a movement from understanding what the text says to what the text really talks about.

The phenomenological-hermeneutic methodology developed by Lindseth and Norberg ${ }^{26}$ includes the following three stages: 1) naïve reading, which is the phase where the interpreter seeks to grasp an initial understanding of the text as a whole; 2) structural analysis, which includes confirmation of the initial understanding of the naïve reading through several examinations of the parts in the text formulated as themes and subthemes; and 3) comprehensive understanding, which is an in-depth interpretation in which the text is once again read 
as a whole and reflected on in light of the interpreter's preunderstanding, the results emerging from the naïve reading and the structural analysis, and relevant theory and literature to gain a deeper understanding of the text.

\section{Ethics}

The study was approved by Norwegian Social Science Data Services on April 17, 2012. All participants were given written information about the study, which was repeated orally before the interview started. Confidentiality and the voluntary nature of participation in the study were assured, ${ }^{29}$ as well as the participants' right to withdraw from the study at any time if they wanted. Written informed consent was obtained from each participant before the study began.

\section{Results}

\section{Naïve reading}

Being a home nursing leader and responsible for facilitating a good transition process for older care-dependent people after a hospital stay represents several challenges. These challenges include the organization of services and the cooperation, communication, and clarification of responsibility between the different organizational levels and the parts involved. Being in charge of the home nursing services is perceived to be stimulating and enriching. However, it also involves continually being forced to make tough prioritizations and to balance several competing and contradictory interests. Fundamental ideals and values in nursing and nursing leadership for providing holistic, individualized, and excellent patient care are threatened by the increased demands for efficiency in times of limited personnel resources. Being a home nursing leader also includes balancing the patient's need for continuity, the division of the "right" professional competence in specific care situations, and the staff need for breaks to prevent burnout.

\section{Structural analysis}

Three themes and seven subthemes emerged from the structural analysis of the text, reflecting the meaning of the nursing leaders' abilities to provide quality care in the patients' transition processes from hospital to a home care setting. An overview of the themes and subthemes are presented in Table 1. Subsequently, they are deepened and described in the text that follows.

\section{Challenged by the organization of home care services}

Factors related to the municipal organization of services, and the fact that decisions about care arrangements and delivery were made by an external unit, were major concerns. These factors represented great challenges, particularly regarding patients in transition.

\section{Lack of involvement in the transitional process}

The responsibility for deciding and planning home care after discharge from hospital was placed on the purchaser unit. Consequently, the patients often returned to their homes without home care services being involved. The participants expressed frustration and worries about this praxis, which left them uninformed and unprepared. They also experienced lack of routine and a forum for good communication with the hospital wards. Subsequently, this affected the home nursing leaders' abilities to provide a smooth transition process. In addition, they indicated that often patients were discharged late on a Friday afternoon, sometimes without any case summary, care plan, or prescribed medication, and without being able to obtain necessary technical aids. The participants described this as particularly unfortunate. This left the leaders in a difficult situation, and their relationship and cooperation with the patient and their next of kin got off to an unfortunate start. One of the informants expressed it this way:

It is all about planning! Sometimes everything happens so fast and the situation may seem very chaotic and difficult to grasp. They [the hospital staff] call us 1 or 2 hours before the patient is sent home, often on Friday before a weekend, and we have no chance to prepare for a good homecoming and provide for sufficient competent care personnel. But you know, in a way ... we just have to say "thank you" ...

Table I Overview of the themes and subthemes

\begin{tabular}{llll}
\hline Themes & $\begin{array}{l}\text { Challenged by the organization } \\
\text { of home care services }\end{array}$ & $\begin{array}{l}\text { Acting in a shifting and unsettled } \\
\text { context }\end{array}$ & $\begin{array}{l}\text { Endeavored to deliver care in } \\
\text { accordance with professional values }\end{array}$ \\
\hline Subthemes & Lack of involvement in the transitional & Adjusting internal resources to \\
process & $\begin{array}{l}\text { external demands and expectations } \\
\text { Administration of care decided at } \\
\text { another level }\end{array}$ & $\begin{array}{l}\text { Providing for appropriate internal } \\
\text { systems and routines }\end{array}$ \\
& & & $\begin{array}{l}\text { Prioritizing professional competence } \\
\end{array}$ \\
\end{tabular}


and take what comes. And so we do, we do what we can to provide the care and help they need.

The participants expressed frustration about the lack of understanding they felt that other instances had regarding the home nurses' work situation. They felt that the hospital staff and that the purchaser unit thought they could handle every matter immediately. They also perceived that this way of organizing health services decreased the quality of care.

\section{Administration of care decided at another level}

Another challenge described by the home nursing leaders was the quality of the assessment and decisions regarding the individual patient's care needs. This challenge was linked to the purchaser-provider model to which all the participants had to relate. They claimed that the organizational system left the home nursing unit - that is, the provider unit - with little power and influence on the care planning for the individual patient. The participants described several problems and frustrations in this respect. Often they had to start providing care to the patient before assessors from the purchaser team had made their home visit. Sometimes the purchasers even made the assessment by telephone. In these cases, the home nursing leaders felt that the care arrangement appeared temporary and improvisatory. One of them commented:

On our first visit to the patient, we are supposed to go through and discuss the resolution made by the purchaser unit, but this is not possible because they haven't been there yet. A bad start, if you ask me. They haven't had time ... and so they do it on the phone ... how can you map the patient's total situation and needs without meeting him?

The participants also had a distinct impression that the purchasers were very task and procedure oriented. The participants often appeared to be unable to make appropriate decisions because they lacked insight into the complete situation of the patient and their family. In that sense, some of the participants missed "the good old days" when the home nursing staff was responsible for making the assessment and decisions:

In previous times, the responsibility for making the first home visit and assessment was placed on the home nurses who also were the ones responsible for the care provision.

I think this was so much better ... we are so used to a holistic way of thinking and we have so much experience from working in the patient's home.

Many of the participants clearly expressed skepticism regarding the quality of the needs assessment and care planning. They also experienced it as unnecessarily bureaucratic and time consuming. They often felt that the decisions were incomplete and that they corresponded to the patients' needs to a very limited degree. As a consequence, they had to do the assessment again. In their opinion, this reassessment was fundamental to achieving the provision of quality care to patients in their home after hospital discharge. It was the home nursing leaders who were responsible for the day-to-day care of the patients, and it was them who had to face the criticism and complaints if the patients or their families were dissatisfied with the care arrangements. Some of the participants also described feelings of being ignored and degraded and that their competence and experience were only utilized to a limited extent in the current system.

Conclusively, the nursing leaders not only described the system as a limiting factor for good quality care provision in the actual transition phase but also expressed many concerns about the decisions or resolutions made. Some of the leaders expressed that they felt relieved, in a way, for not being held responsible for making wrong decisions, as the responsibility for decision making was placed on another unit. This made it possible to refer to and blame the purchaser unit if anything went wrong in the initial phase of the patient's transition process.

\section{Acting in a shifting and unsettled context}

The rapid and frequent changes in the health care system during the last years, together with decreasing resources and increasing workload, challenged the home nursing leaders. This caused a continuous need for tough prioritizing. At the same time, they realized the significance of assuring quality care provided by competent and ethical reflective personnel.

\section{Adjusting internal resources to external demands and expectations}

All the participants talked a lot about the pressure they felt due to the imbalance between demands and available resources. The introduction of the Coordination Reform led to rapid discharge of patients from hospital and resulted in a distinct increase in older people who returned to their homes with complex health conditions and in need of advanced nursing care and treatment. The situation involved new challenges for the leaders, as the home nurses had to deal with new tasks that they were unfamiliar with; for example, patients who needed advanced medical treatment and technological equipment. They often lacked training in performing such tasks, and had insufficient time to prepare and obtain the 
required skills. These situations could easily result in very unfortunate situations in which the patients and their relatives, as well as the nurses, felt uncomfortable and unsecure. In addition, the home nursing leaders were concerned about limitations and lack of required precautions when certain procedures had to be performed in the patients' homes. One of the participants gave this example:

Giving patients' intravenous fluids and nutrition is not a problem, but often they need specific medications such as antibiotics and you know we do not have necessary environmental equipment like [an] exhaust room and so on, which is a matter of course in hospital.

Thus, the leaders felt that the expectations from external parties, particularly from the hospital and the purchaser unit, were unrealistic. In their opinion, they lacked insight into the work situation in the home care services and showed little understanding for the limitations inherent in providing nursing care in home surroundings.

\section{Experiencing lack of communication}

with significant others

The participants expressed many concerns related to the consequences they experienced caused by insufficient communication. They maintained that the flow and quality of communication between the different professionals involved in the patients' discharge processes was a decisive aspect for the arrangement and provision of quality care. They often experienced the communication between the hospital and home nursing unit as insufficient or even lacking. In addition, the interdisciplinary communication and cooperation on the municipal level was described as unsatisfactory and complicated. This was related to the organization of care services, as described earlier, but they also experienced unsatisfactory cooperation between professionals when they were involved in the care of the same patient. A particularly vulnerable patient group mentioned by several of the participants was that of patients with psychiatric problems. In these cases, the patients often needed coordinated care services that involved follow-up by nurses from the psychiatric unit as well as general care and attention from the home nurses. The participants perceived that these situations could be problematic and frustrating because they felt ignored by the psychiatric nurses who were unwilling to share their knowledge about the patients. One of them expressed it this way:

The psychiatric staff is somewhat sovereign you know, they do what they want! They are not particularly willing to cooperate and make appointments. They are the ones most difficult to cooperate with, and I feel that it is so unfortunate because these patients are a particularly vulnerable group. It seems like they have no understanding for what we actually do ... and neither have they any questions ... they are arrogant!

Another important aspect described by almost all of the leaders was the significance of proper involvement and communication with the patient's next of kin, whether they lived with the patient or not. Next of kin were regarded as an inestimable resource for home-living older persons, and cooperation with them in the transition process was seen as decisive when planning for a good care arrangement. Sometimes their needs and wishes differed from those of the patients, and sometimes they had unrealistic expectations about the possibilities for care provision. As one of the participants explained:

It may be a challenge when the next of kin perceives that the patient's needs and situation are more severe than the patient himself views it. In these situations, it is particularly important to clarify expectations and facilitate for care arrangements in agreement with all parties involved.

However, next of kin were often the ones who knew the individual patient's situation the best and, thus, represented a rich information source for the home nurses:

It is so important to work in close cooperation with the patient's next of kin, particularly when the patient is cognitively declined or suffers from dementia. When the patient is not able to express himself, the next of kin is the most valuable resource to get an insight into his life and care needs.

Consequently, the leaders highly emphasized involvement, communication, and cooperation with the next of kin. Further, they endeavored to plan and make the transition process as predictable as possible for the patient as well as their close relatives. In many cases, the next of kin acted as informal care providers, and the division of care between the informal and professional care providers needed to be settled.

\section{Endeavored to deliver care in accordance with professional values}

The challenges faced by the participants in this study were, to a great extent, related to organizational aspects on different levels and units within the health care system. However, the participants were also concerned about challenges related to their own unit and their professional values. They 
acknowledged their responsibilities as leaders to make sure that the care they delivered to the patients was of good quality. In this respect, they described several important issues.

\section{Providing for appropriate internal systems and routines}

Having sufficient and well-run internal systems and predictable routines was emphasized by most of the participants as an important success criterion for providing quality care for patients in the transition process. They described many challenges related to staff shortages and lack of continuity in patient care resulting from many part-time and temporary employees. Due to these staff-related challenges, it was of utmost importance to have internal systems that were updated and predictable:

Unfortunately, there are so many persons involved in the care of each patient, and of course, this makes the system particularly vulnerable, and thoroughly updated documentation is so important.

All participants had ambitions that the documentation and care plans should be functional and updated. They also emphasized the necessity of having well-written, informative, and easily available practice procedures, as well as updated and appropriately composed patient lists. Thoroughly written care plans were particularly important when the patient had been in hospital for some time and suffered from complex or serious health conditions or dementia. These patient groups were considered especially vulnerable and in need of wellprepared and -planned advanced holistic care provided by competent professionals. In addition, it was felt that these patients had often been through a tough hospital stay and should be protected from unnecessary burdens caused by a nonfunctioning system or insufficient documentation.

\section{Prioritizing professional competence}

Although primary nursing and continuity in patient care were the goals preferred by all participants, they also described that these goals were quite unrealistic. They acknowledged the need for improved professional competences, as the care situations the home nurses had to handle were increasingly complex. At the same time, the shortage of qualified and trained nurses forced them to make tough decisions about priorities concerning available resources. However, the consequences of such prioritization often resulted in further concerns, such as the need for professional qualifications for performing certain tasks at the expense of continuity of care. Holding this responsibility was experienced as both difficult and frustrating. One of the participants expressed:
Although I try to facilitate for continuity I often fail in this sense, simply because I have to prioritize some patients' need for specific treatment or follow-up at the expense of continuity for all. In the transition process, it is particularly important to prioritize enough and proper professional competence.

The participants also described another aspect of prioritizing professional competence. To keep up with the demand for increased professional and advanced competence, the leaders emphasized and facilitated regular courses and training. Although they did not always succeed, they considered this an important responsibility to ensure good quality care. Their responsibility also included how the individual staff member perceived their job satisfaction. Care situations that were very intensive and demanding represented a potential risk for burnout among staff members. Therefore, the leaders' responsibilities and priorities included concerns regarding staff members in addition to patient care.

\section{Maintaining a professional culture}

Most of the participants highlighted the importance of the quality of the internal culture and atmosphere in their unit. They described how they strived to facilitate an open and supporting climate, allowing everyone in their personnel group to be seen and heard, and to express and discuss problematic issues. Many of them had allocated fixed time for discussing ethical problems. Dilemmas related to patient situations as well as reflections on their own attitudes and values were regularly on the schedule. Some of the leaders described that certain values and principles were inherent in the culture, such as striving for the patients' independence, self-care, and giving "help for self-help," or striving to provide individual and holistic care to everyone. They talked about how valuable these discussions and reflections were for maintaining a professional culture characterized by quality, solidarity, and team spirit. They felt they had a major responsibility as leaders to encourage and support staff members to maintain enthusiasm and engagement - particularly in times of tough pressure, personnel shortage, and heavy workload. This was also crucial for quality of care in general, and for patients in transition from hospital to home in particular. They argued that the quality of care in this phase could be decisive for future patient-nurse relationships.

\section{Discussion Comprehensive understanding and reflections}

The nursing leaders experienced several challenges, contradictory interests, and conflicts in their efforts to assure that 
older patients underwent a healthy transition between hospital and community home care services. These challenges were related to the organization of the care services and the unsatisfactory cooperation between different care levels in the health care services and between units in the municipality. Further, they were challenged by the imbalance between demands for improved efficiency and competence and the actual, available resources. This represented a threat to the quality of care and the leaders' professional and ethical values. Therefore, the organization of quality care depended on the nursing leaders' ability to adjust and act with flexibility and pragmatism.

Problems related to transitions between levels of care, resulting in unmet care needs and extended health costs, have been outlined in several studies. ${ }^{11,30,31}$ These problems can be associated with a lack of coordinated services and are also described as major challenges in the Norwegian health services. ${ }^{3}$ Insufficient patient information being communicated between the health care levels is one such problem that was also described by the participants in our study. The possible consequences were severe for the patients as well as for the health care providers. Meeting the older patients' information needs is recognized as a crucial factor in the patients' successful integration into their prior living environment after hospital discharge. Unmet information needs have also been shown to increase the risk of readmission. ${ }^{32}$ Older patients in transition from hospital to home are often frail and have severe and complex health conditions. They are often exposed to speedy readmission and their communication and information needs might be considerable. To meet these needs, it is extremely important that the home care services receive critical information about the patient in a timely manner. The exchange of critical information about the patient was highlighted by the participants in our study as a fundamental aspect for establishing a trusting relationship with the patient and next of kin, and for providing appropriate advanced nursing care. In this respect, the communication flow between the two levels of care is decisive.

Communication flow between the two organizational levels has been described as a critical prerequisite for accomplishing an appropriate and smooth transition process for older persons when they are discharged from hospital to the community and in need of home nursing. ${ }^{14}$ Our participants found that the lack of routine and a forum for communication with the hospital wards affected their ability to facilitate a smooth transition process. The need for improved communication between hospital health professionals and the municipal health sector has also been described in other studies, ${ }^{14,16}$ and the lack of communication and inappropriate routines appear to be critical factors in patient transfers and transitions between these levels. According to Coleman and Berenson, ${ }^{11}$ one explanation for the existing problem is that many of the professionals involved in transitional care have never practiced in the context to which they are transferring the patients. Better knowledge and insight into each other's working context might be an unpretentious, although effective, first ambition for detecting communication gaps. This could also be an important basis for working out functional guidelines and communication systems between the settings. According to Dunnion and Kelly, ${ }^{14}$ the importance of such systems cannot be over emphasized, particularly when a vulnerable group is involved.

Home nursing and home nursing leaders are parts of a context that has moved toward economic rationalism. The number of available hospital beds is reduced, the length of hospital stays has decreased, and the focus on efficiency and cost containment is noticeable. ${ }^{16}$ Consequently, the pressure on nursing leaders in hospital wards as well as on home nursing leaders has increased. A dual understanding of how this pressure may affect older patients in transition from hospital to home is fundamental for identifying the challenges this specific group of exposed patients face. Shared visions of opportunities for quality improvement may result in a reduction of common problems in transitions between settings, such as limited clinical information and errors related to medications. ${ }^{6}$ It is assumed that improved cooperation and coordination between nursing leaders in hospitals and home care services will contribute to a decrease in readmissions, which has been reported as a major problem. ${ }^{4,5,17}$

According to the findings of the current study, the purchaser-provider model may have some advantages including assuring justice for the patients and transparency in care delivery. Vab $\varnothing^{12}$ points out that this organization of services may be more predictable and the procedures for decision making more correct. The model has the potential to assign more resources. Nevertheless, Vab $\varnothing^{33}$ also defines a number of disadvantages related to the same model, which are in agreement with the experiences described by the participants in our study. These disadvantages were connected to their concerns about providing care that was assessed and planned by an external unit. Care decisions made by the purchaser unit were often perceived as incomplete and inappropriate. It therefore seemed that the purchaser-provider model put the quality of care at stake.

The home nursing leaders in our study made their own priorities and flexible adjustments in relation to the individual patient's condition. This was necessary to maintain 
professional values and provide excellent care even in a challenging patient situation. Similar findings have been reported by Wollscheid et al..$^{34}$ Different pragmatic strategies are used by the leaders when needed. To maintain professional values and ethical norms in the provision of quality care appeared to be more important than written obligations carried out by the purchaser unit. The incongruence between professional values and organizational values create ethical conflicts in which the nurses' moral integrity is challenged. ${ }^{35}$ When facing these ethical conflicts, they may end up compromising their moral integrity for the sake of the organization.

Our findings revealed that the demand for highly competent professionals in the home nursing services has increased. At the same time, care personnel working in the community are, in general, less educated and qualified to perform advanced medical and nursing care than their hospital-employed counterparts. ${ }^{36}$ This reality was also reflected by the participants in our study. Regularly they had to deal with the imbalance between demands and available resources. They were forced to find solutions to ensure that the patients with the most complex conditions were cared for by the most qualified professionals. However, they were aware of the undesirable consequences this could have for care continuity, which was viewed as a crucial component for providing home care of high quality. ${ }^{37}$ The lack of continuity may be particularly unfortunate for vulnerable patients in transition from hospital to home. This also indicates that nursing leaders in the home care services are forced to compromise and prioritize between the ideal of continuity in care and the real day-to-day work situation.

A major ambition of the Coordination Reform is to make the health care system more efficient. The reform relies heavily on economic incentives, as much of the cost is now transferred from the hospitals to the communities..$^{36}$ This adds several dilemmas to the nursing leaders in the home care services. The Norwegian philosopher Vetlesen ${ }^{13}$ claims that the demands for health service efficiency challenge professional and moral integrity, which was also indicated in our study. According to Vetlesen, ${ }^{13}$ the alternatives are either to take the burdens of making an effort to obtain the dictated ambitions or to take the burdens of not obtaining them. The nursing leaders in our study seemed to balance the contradictory interests by using their own strategies based on flexibility and pragmatism in an effort to maintain quality care.

\section{Methodological considerations}

The present interpretation of the interview texts is only one among many possible interpretations. Due to the specific nature of a qualitative study, it is not realistic to discuss issues of validity and reliability or to generalize. However, despite limitations related to the sample size and diversity, many of the aspects described by the participants correspond to findings in other studies. Thus, it is reasonable to assume that the challenges revealed in the findings are transferable to nursing leaders working in similar contexts. The organization and funding of care services differ greatly between countries and cultures, but the new public management movement has influenced organizations worldwide. Therefore, we assume that most of the findings in the current study are readily recognizable. The interview texts provided rich descriptions of what it means to be a nursing leader who holds the responsibility for providing home care of high quality to older people in transition from hospital to their own home, and similarities as well as variations in the studied phenomenon were discovered.

\section{Conclusion}

Home nursing leaders have to deal with complex and multifactorial responsibilities related to the system and organizational levels. In addition, they have to handle challenges related to care providers, patients, and provision of care. This study revealed that the nursing leaders who participated faced several barriers in the administration of quality care to older patients in transition from hospital to their homes. To overcome the barriers, they were flexible and pragmatic and seemed to accept an individual responsibility as leaders for delivering care of high quality. This responsibility often involved professional and ethical dilemmas. By accepting this individual responsibility, one may ask if they simultaneously, in a paradoxically and unintended way, contributed to undermining their own professional ideals. This might be an under-recognized issue that has received too little attention. Nevertheless, healthy transitions are crucial for patient satisfaction and safety. Healthy transitions also have economic incentives. Providing care of high quality is critical and, thus, individual responsibility and interventions are necessary. However, it is also of utmost importance to go beyond individual responsibility and explore factors at the system level to address how these multiple and complex factors affect the quality of care to older patients in the transition from hospital to home care services.

Further research is needed to identify how best practice related to transitional care can be administered in the home care services as well as in the hospitals. In this respect, both qualitative and quantitative studies should be welcomed. More studies are needed to obtain insight into the purchaser 
unit and its involvement in the transition process. Studies focusing on the persons directly involved in the transition process - namely, the older patients and their next of kin would also be valuable.

\section{Acknowledgments}

The authors would like to thank all the home nursing leaders who participated and so generously shared their experiences and views in this study.

The study was financially supported by the Faculty of Health and Sport Sciences, University of Agder, Norway, and Faculty of Health and Social Studies, Telemark University College, Norway.

\section{Disclosure}

The authors declare no conflicts of interest in this work.

\section{References}

1. Naylor MD. Transitional care: a critical dimension of the home healthcare quality agenda. J Healthc Qual. 2006;28(1):20-28.

2. Naylor MD. Advancing high value transitional care: the central role of nursing and its leadership. Nurs Adm Q. 2012;36(2):115-126.

3. Norwegian Ministry of Health and Care Services. The Coordination Reform: Proper Treatment - At the Right Place and Right Time. Report No 47 (2009) to the Storting. Oslo: Department of Government Publications.

4. Lindman AS, Damgaard K, Tjomsland O, Helgeland J. Reinnleggelser av eldre $i$ Norge [Readmission in Norwegian hospitals]. Oslo: Nasjonalt kunnskapssenter for helsetjenesten (NOKC) [Norwegian Knowledge Centre for the Health Services]; 2012. Norwegian. Available at: http:// www.kunnskapssenteret.no/Publikasjoner/_attachment/15461?_ ts $=13735 \mathrm{c} 2$ dea9 \&download=true. Accessed August 29, 2013.

5. Heggestad T. Do hospital length of stay and staffing ratio affect elderly patients' risk of readmission? A nation-wide study of Norwegian hospitals. Health Serv Res. 2002;37(3):647-665.

6. Boling PA. Care transitions and home health care. Clin Geriatr Med. 2009;25(1):135-148.

7. Naylor MD, Feldman PH, Keating S, Koren MJ, Kurtzman ET, Maccoy MC, Krakauer R. Translating research into practice: transitional care for older adults. J Eval Clin Practice. 2009;15(6):887-1237.

8. Coleman EA, Min SJ, Chomiak A, Kramer AM. Posthospital care transitions: patterns, complications, and risk identification. Health Serv Res. 2004;39(5):1449-1465.

9. Schumacher KL, Jones PS, Meleis AI. Helping elderly persons in transition: a framework for research and practice. In: Swanson EA, Tripp-Reimer T, editors. Life Transitions in the Older Adult: Issues for Nurses and Other Health Professionals. New York, NY: Springer; 1999:1-26.

10. Meleis AI, Trangenstein P. Facilitating transitions: redefinition of the nursing mission. Nurs Outlook. 1994;42(6):255-259.

11. Coleman EA, Berenson RA. Lost in transition: challenges and opportunities for improving the quality of transitional care. Ann Intern Med. 2004;141(7):533-536.

12. Vabø M. Norwegian home care in transition - heading for accountability, off-loading responsibilities. Health Soc Care Community. 2012;20(3):283-291.

13. Vetlesen AJ. Empati under press [Empathy under pressure]. Sykepleien. 2010;3:60-63. Norwegian.

14. Dunnion ME, Kelly B. All referrals completed? The issues of liaison and documentation when discharging older people from an emergency department to home. J Clin Nurs. 2008;17(18):2471-2479.
15. Dedhia P, Kravet S, Bulger J, et al. A quality improvement intervention to facilitate the transition of older adults from three hospitals back to their homes. J Am Geriatr Soc. 2009;57(9):1540-1546.

16. Bauer M, Fitzgerald L, Haesler E, Manfrin M. Hospital discharge planning for frail older people and their family. Are we delivering best practice? A review of the evidence. J Clin Nurs. 2009;18: 2539-2546.

17. Golden AG, Tewary S, Dang S, Roos BA. Care management's challenges and opportunities to reduce the rapid rehospitalization of frail community-dwelling older adults. Gerontologist. 2010;50(4): 451-458.

18. Naylor MD. Transitional care of older adults. Annu Rev Nurs Res. 2002;20:127-147.

19. Naylor MD, Brooten DA, Campbell RL, Maislin G, McCauley KM, Schwartz JS. Transitional care of older adults hospitalized with heart failure: a randomized, controlled trial. J Am Geriatr Soc. 2004;52(5): 675-684.

20. Coleman EA, Parry C, Chalmers S, Min SJ. The care transitions intervention: results of a randomized controlled trial. Arch Intern Med. 2006;166(17):1822-1828.

21. Naylor MD, Aiken LH, Kurtzman ET, Olds DM, Hirschman KB. The care span: The importance of transitional care in achieving health reform. Health Aff (Millwood). 2011;30(4):746-754.

22. Danielsen B, Fjær S. Erfaringer med å overføre syke eldre pasienter fra sykehus til kommune [Experiences related to the transfer of older patients from hospital to municipality]. Sykepleien Forskning. 2010;5(1):28-34. Norwegian.

23. Hvalvik S, Dale B. Nurses' Experiences of Caring for Older Persons in Transition to Receive Homecare: Being Somewhere in between Competing Values. Nurs Res Pract. 2013;2013:181670.

24. Kvale S, Brinkmann S. Det kvalitative forskningsintervju. [The qualitative research interview]. 2nd ed. Oslo: Gyldendal Akademisk; 2009.

25. Polit DF, Beck CT. Essentials of Nursing Research: Appraising Evidence for Nursing Practice. 7th ed. Philadelphia, PA: Lippincott Williams \& Wilkins; 2010.

26. Lindseth A, Norberg A. A phenomenological hermeneutical method for researching lived experience. Scand J Caring Sci. 2004;18(2): $145-153$.

27. Ricoeur P. Interpretation Theory: Discourse and the Surplus of Meaning. Fort Worth, TX: Texas Christian University Press; 1976.

28. Geanellos R. Exploring Ricoeur's hermeneutic theory of interpretation as a method of analysing research texts. Nurs Inq. 2000;7(2):112-119.

29. Beauchamp TL, Childress JF. Principles of Biomedical Ethics. 6th ed. Oxford: Oxford University Press; 2009.

30. Garåsen H. The Trondheim model: improving the professional communication between the various levels of health care services and implementation of intermediate care at a community hospital could provide better care for older patients: short and long term effects [PhD thesis]. Trondheim: Norwegian University of Science and Technology; 2008. Available at: http://ntnu.diva-portal.org/smash/ record.jsf?pid=diva2:123898. Accessed August 29, 2013.

31. Naylor M, Keating SA. Transitional care: moving patients from one care setting to another. Am J Nurs. 2008;108(Suppl 9):58-63.

32. Romagnoli KM, Handler SM, Ligons FM, Hochheiser H. Home-care nurses' perceptions of unmet information needs and communication difficulties of older patients in the immediate post-hospital discharge period. BMJ Qual Saf. 2013;22(4):324-332.

33. Vabø M. Home care in transition: the complex dynamic of competing drivers of change in Norway. J Health Org Man. 2009;23(3): 346-359.

34. Wollscheid S, Eriksen J, Hallvik J. Undermining the rules in home care services for the elderly in Norway: flexibility and cooperation. Scand J Caring Sci. 2013;27(2):414-421.

35. Hendel T, Steinman M. Israeli nurse managers' organizational values in today's health care environment. Nurs Ethics. 2002;9(6): 651-662. 
36. Romøren TI, Torjesen DO, Landmark B. Promoting coordination in Norwegian health care. Int J Integr Care. 2011;11(Spec 10th Anniversary Ed):e127.
37. Gjevjon ER, Romøren TI, Kjøs BØ, Hellesø R. Continuity of care in home health-care practice: two management paradoxes. J Nurs Manage. 2013;21(1):182-190.

\section{Publish your work in this journal}

The Journal of Multidisciplinary Healthcare is an international, peerreviewed open-access journal that aims to represent and publish research in healthcare areas delivered by practitioners of different disciplines. This includes studies and reviews conducted by multidisciplinary teams as well as research which evaluates the results or conduct of such teams or health- care processes in general. The journal covers a wide range of areas and welcomes submission from practitioners at all levels, from all over the world. The manuscript management system is completely online and includes a very quick and fair peer-review system. Visit http://www.dovepress. com/testimonials.php to read real quotes from published authors.

Submit your manuscript here: http://www.dovepress.com/journal-of-multidisciplinary-healthcare-journal 